\title{
De las libertades de Internet al tráfico: evolución del debate en torno a la neutralidad de la red
}

\section{From Internet freedoms to traffic management: evolution of the debate on network neutrality}

\author{
Andreu Castellet Homet \\ Profesor de Comunicación Audiovisual \\ Departamento de Información y Documentación \\ (Universidad de Murcia) \\ Juan Miguel Aguado Terrón \\ Profesor titular de Teoría de la Comunicación \\ Departamento de Información y Documentación \\ (Universidad de Murcia) \\ Inmaculada José Martínez Martínez \\ Profesora titular de Publicidad \\ Departamento de Información y Documentación \\ (Universidad de Murcia)
}

Fecha de recepción: 12 de julio de 2013

Fecha de revisión: 24 de enero de 2014

Para citar este artículo: Castellet Homet, A., Aguado Terrón, J.M. y Martínez Martínez, I.J. (2014): De las libertades de Internet al tráfico: evolución del debate en torno a la neutralidad de la red, Icono 14, volumen (12), pp. 431-461. doi: 10.7195/ri14.v12i1.613 


\section{Resumen}

La neutralidad de Internet es uno de los asuntos más debatidos a la hora de diseñar las políticas públicas en relación con la red. Del modo en que este se va decantando depende crucialmente la preservación de los derechos de los usuarios en relación con la red de redes, tanto en su versión fija como en la móvil. De ahí su importancia para todos los sectores vinculados con los contenidos digitales. El trabajo analiza cómo las autoridades responsables han enfocado los problemas de la neutralidad en Estados Unidos, Japón y la Unión Europea, con énfasis especial en la evolución del discurso de las autoridades en relación con el asunto. Los autores concluyen que los poderes públicos han concentrado la mayor parte de su esfuerzo en la cuestión de la regulación del tráfico, en detrimento de otros aspectos no menos relevantes.

\section{Palabras clave}

Neutralidad de la red - Internet - Contenidos digitales - Internet móvil

\section{Abstract}

Internet neutrality is among the most controversial issues when it comes to the design of media and Internet policies. The way the dispute is resolved will shape the way rights of users' access to any kind of content will be preserved. Hence its importance to all sectors related to digital production and distribution of media. Telecommunications companies, content producers and app developers, digital media platforms, users and regulators of all kinds, are the main actors involved. This paper analyses how policy makers have focused from different points of view on network neutrality in the United States, Japan and the European Union, in both fixed and mobile networks, with special emphasis on the evolution of the public debate on the different angles of the matter. The authors conclude that the authorities have concentrated most of their efforts on the issue of traffic regulation at the expense of other equally important aspects.

\section{Key Words}

Internet governance - Network neutrality - Mobile Internet - Internet regulation Digital media - Media policies

ICONO14 | Año 2014 Volumen 12 NN $^{\circ}$ | ISSN: 1697-8293 | DOI: ri14.v12i1.613 


\section{Introducción}

La extensión del uso de la red de banda ancha a la mayor parte de la población y el desarrollo de servicios basados en ella han puesto de relieve la cuestión de la neutralidad de la red. La popularización de los dispositivos móviles -smartphones, tablets y ciberlibros, entre otros- y el consiguiente crecimiento del flujo de datos han dado lugar a un conjunto de aplicaciones y servicios en la red concebidos específicamente para estos terminales, con lo que puede hablarse con propiedad de un Internet móvil con características específicas derivadas del acceso en movilidad.

Así, la cuestión de la neutralidad de la red adquiere un relieve creciente y pone en primer plano la cuestión de su preservación. Los puntos de vista más convencionales, que habían sostenido que se trataba de una cuestión meramente académica, sin sustento en una secuencia de casos prácticos, han sido desbordados por la realidad. Los reguladores europeos admiten que al menos un $20 \%$ de los usuarios de Internet móvil en la Unión Europea (UE) experimentan restricciones en su uso de la red (BEREC, 2012a). Aunque solo han podido documentarse en esta proporción las prácticas de bloqueo del acceso a servicios de voz IP (VoIP), que eluden las tarifas de voz de los operadores, esto solo ha podido demostrarse gracias al empeño de las compañías que prestan estos servicios, que han apoyado activamente a sus usuarios y han participado en las encuestas públicas sobre la neutralidad. Por todo ello es razonable pensar que otro tipo de vulneraciones de la libertad del usuario en lo que respecta al acceso a contenidos -video y audio en streaming, servicios de descargas P2P-podrían estar sucediendo por acción de algunos operadores sin que los usuarios fueran capaces de atribuir sus dificultades a la gestión de la red.

En plena fase de expansión de Internet por todo el mundo, acompañada por el fuerte crecimiento del consumo de contenidos móviles, ya sea en su versión más convencional o en la de aplicaciones/servicios, se hace necesario someter a examen la acción reguladora, encargada de velar por la efectividad de la protección de los derechos ciudadanos en relación con su libertad de acceso y de producción de información de todo tipo. Como señalan Castellet y Aguado, “con ser muy im- 
portante a los efectos del debate sobre libertades públicas, el de la neutralidad resulta igualmente relevante cuando se trata de su capacidad para determinar la estructura del negocio de los contenidos digitales" (2010, p. 4).

En este artículo se presta especial atención al énfasis de los reguladores sobre los diferentes aspectos de la neutralidad, con la pretensión de resaltar coincidencias, divergencias y correlaciones entre los actos de los dos mayores reguladores estudiados -la FCC y la UE-, y de relatar en qué medida en impacto del desarrollo de Internet móvil ha influido en el discurso regulador sobre la neutralidad. El caso japonés se menciona como muestra de un modo diferente de orientar las políticas públicas sobre Internet, aunque se analiza con menor detalle en la medida en que sus acciones se desarrollan con instrumentos más informales, basados a menudo en el consenso, y por lo tanto más difíciles de comparar con los occidentales.

Al citado análisis de la políticas sobre la neutralidad de Internet pretende contribuir el presente trabajo, que se estructura del siguiente modo: tras la introducción se expone el método empleado y los materiales utilizados; a continuación se sitúa el debate sobre el concepto de neutralidad a la luz de la evolución histórica de la red desde su creación; se repasan los principales hitos reguladores en La UE y EEUU, y se examina el estado de la cuestión en el mercado japonés; y se comparan las acciones reguladoras emprendidas en los tres mercados, con una atención especial en la comparación UE-EEUU. Por último, se enuncian las conclusiones alcanzadas y los vectores más relevantes de discusión apreciados.

\section{Metodología}

Para la elaboración de este trabajo se ha estudiado la evolución de las políticas públicas sobre la neutralidad de Internet en los tres mayores mercados abiertos ${ }^{1}$ del mundo: Estados Unidos (EEUU), Japón y la UE, con una atención principal sobre la realidad española. Para ello se han examinado las resoluciones más relevantes promulgadas en cada uno de los tres mercados (la Federal Communications Commission (FCC) en EEUU; la Ley del Negocio de las Telecomunicaciones japonesa; y en la UE, declaraciones y resoluciones del Parlamento Europeo, la Comisión Europea (CE), el organismo regulador de las comunicaciones europeas (BEREC), y la Comisión del Mercado de las Telecomu-

ICONO14 | Año 2014 Volumen $12 N^{\circ} 1$ | ISSN: 1697-8293 | DOl: ri14.v12i1.613 
nicaciones (CMT) de España. También se ha recurrido a la revisión de lo más relevante de la abundante literatura académica y profesional y de otros reguladores nacionales e internacionales sobre la neutralidad (ARCEP, 2010; Benkler, 2011; Berners-Lee, 2006; Castellet y Aguado, 2010; Cheng, Bandyopdhyay, y Guo, 2011; Cisco, 2010; Carter, Marcus, Peake, y Watanabe, 2010; Copps, 2003; Clyburn, 2010; Gamo, 2007; Hustinx, 2011; ITU, 2012; Jaokar y Gatti, 2009; Lessig, 1999, 2009,; Murakami, 2007; Noam, 2001, 2010; Odlyzko, 2009; Ofcom, 2011; Vesa, 2007; Wu, 2004, 2011). Todo ello ha precisado ser contrastado con el curso efectivo de las decisiones políticas y de las reacciones de los diferentes grupos de interés mediante el seguimiento de la actualidad.

Se trata de un asunto que se desenvuelve en un contexto tecnoeconómico, marcado por la interacción de empresas y consumidores, en el que "intervienen decisiones tácticas pero también requieren diferentes avances tecnológicos, claramente más complejos [y no siempre disponibles]" (Feijóo, Gómez-Barroso y Martínez, 2010, p. 142). Pero también se hace necesario incorporar en lugar preferente el examen de los diversos desarrollos regulatorios, de diferente naturaleza en cada espacio de mercado, por lo que la perspectiva interdisciplinar viene exigida por las características de nuestro objeto de estudio. No en vano la aportación a la comprensión de la circulación de contenidos en la red se debe también en gran parte a aportaciones procedentes de la ciencia jurídica (Benkler, 2007, y 2010; Lessig, 2009; $\mathrm{Wu}, 2011)$. Esta visión necesariamente poliédrica de la cuestión, con el recurso a datos y a análisis enraizados en disciplinas diferentes de los estudios de comunicación, cuenta con un amplio respaldo en trabajos de académicos especializados en el mundo de la comunicación de masas y el periodismo (Bustamante, 2011; Crusafon Baqués, 2012; Vaidhyanathan, 2011).

\section{Resultados}

\subsection{Definiciones y perspectivas sobre neutralidad de redes}

La gestión de la circulación de los paquetes de información está presente desde los inicios de Internet. La evolución de la red ha desplazado la cuestión desde planteamientos estrictamente técnicos hasta centrar la discusión hacia sus aspectos regulatorios, económicos y políticos.

DOI: ri14.v12i1.613 | ISSN: 1697-8293 | Año 2014 Volumen 12 Nº 1 | ICONO14 
La creación de internet se remite a la puesta en marcha en EEUU en 1958 del proyecto ARPA (Advanced Research Projects Agency) NET. Dicho proyecto fue una de las respuestas a la demostración de poderío tecnológico de la Unión Soviética que supuso el lanzamiento del Sputnik, el primer satélite artificial capaz de orbitar en torno a la tierra. Como señala Fransman (2000, p. 31), el objeto del proyecto de ARPA era la conexión de los ordenadores de gran capacidad entonces existentes mediante la red telefónica ordinaria. Dicho objetivo se consiguió por primera vez en 1969.

Se entiende que internet es una red neutral porque, como señalan Jaokar y Gatti, “todos los paquetes son creados iguales (...). En otras palabras, la red es tonta y la inteligencia se desplaza hacia los extremos de la red (es decir, los nodos y las aplicaciones)" (2009, p. 41). Esta definición se encuadra en la concepción de la red como dumb pipe, o tubería muda, que no interviene en el tránsito de información. Expresado de forma parecida por uno de los creadores del protocolo IP, en el que se ha basado lo que hoy conocemos por internet, "si pago por conectar a la red con una cierta calidad de servicio, y tú pagas por conectar con esa o mayor calidad de servicio, entonces podemos comunicarnos a ese nivel. Es cosa de los proveedores de acceso asegurarse de que interoperan de modo que así suceda" (Berners-Lee, 2006).

Es el de la neutralidad un debate extremadamente polémico, tal vez se trata del aspecto más polémico de la regulación de las actividades en el ciberespacio, hasta el punto de que se discuten los aspectos más básicos que se remontan a los inicios de la red. Basta para ello con comprobar cómo una afirmación tan aparentemente técnica como la formulada por Berners-Lee más arriba encuentra la discrepancia frontal desde un documento del Departamento de Política Científica del Parlamento Europeo: "algunos han sostenido que el protocolo de Internet pretendía desde el principio tratar igual a todo el tráfico, sin preferencia ni priorización. Ello es simplemente incorrecto: la entrega priorizada fue siempre considerada como parte del protocolo de Internet" (Parlamento Europeo, 5/2011).

La necesidad de mantener la red "neutral" es, para Wu, el mantenimiento de "un Internet que no favorezca a una aplicación sobre otras" (2004: 198). 
A estas aproximaciones al concepto de neutralidad de la red merece la pena agregar la elaborada desde la oficina del Supervisor Europeo de Protección de Datos por su capacidad para sintetizar los diferentes aspectos en liza: “la información en Internet debe ser transmitida imparcialmente, sin consideración a su contenido, destino o procedencia (...). Ello significa que los proveedores del servicio de Internet no pueden, a su antojo, priorizar o ralentizar el acceso a ciertas aplicaciones 0 servicios tales como las descargas entre particulares (P2P), etc."(Hustinx, 2011:2).

Al mismo tiempo, la convergencia digital ha alterado la esencia de las empresas operadoras de redes y servicios de telecomunicaciones, inmersas en una transición desde un modelo de ingresos basado en los servicios ligados a la voz a un nuevo modelo "datacéntrico" (Vesa, 2006) en el que la voz está pasando a ser un ingrediente más en un ecosistema de paquetes de datos binarios.

\subsection{Regulación en doble convergencia}

Internet se ha desarrollado desde el principio sobre la base de una paradoja, que combina la máxima libertad de circulación con la regulación. Así lo expresa el creador de la WWW: "pues sí, la regulación para mantener Internet abierto es regulación. Y principalmente, Internet florece a partir de la falta de regulación. Pero hay que preservar ciertos valores básicos" (Berners-Lee, 2006).

La idea de regular no remite exclusivamente a la imposición de normas. Lessig (1999). identifica cuatro modalidades de regulación de conductas en el espacio real y en el ciberespacio Estas modalidades son: la ley, las normas sociales, el mercado, y la arquitectura. En su visión, Lessig entiende que estas cuatro modalidades no se manifiestan de un modo aislado, sino que interactúan entre si, y esta interacción entre modalidades, y las pugnas de unas por imponerse sobre otras quedarán aquí reflejadas.

En el caso español, la regulación tiene un carácter doblemente convergente: por la naturaleza convergente de los contenidos; y porque se desarrolla en un espacio donde se solapan normas y reguladores de diferente alcance, desde las comunidades autónomas hasta la globalidad de la gobernanza de internet, pasan-

DOI: ri14.v12i1.613 | ISSN: 1697-8293 | Año 2014 Volumen 12 Nº 1 | ICONO14 
do por el gobierno de España y la Unión Europea (UE). Castells (2009) define tres áreas principales en las que se manifiesta la regulación de la comunicación: "la regulación del contenido, que incluye la salvaguarda de los derechos de propiedad intelectual, la regulación de la propiedad y la regulación de los servicios impuesta a operadores y emisoras (p. 146). Nuestro ámbito se circunscribe al modo en que la neutralidad de la red incide en el acceso a los contenidos, por lo que la regulación de la propiedad queda al margen de nuestra indagación, y concentraremos nuestra atención principalmente en la regulación de los servicios, que posibilitan el acceso y la circulación de información.

Las controversias sobre la regulación de la red por los poderes públicos comienzan por las dudas acerca de su virtualidad práctica. Como raramente la norma se anticipa a la realidad, la viabilidad de la acción reguladora se pone en cuestión por la evolución acelerada de la arquitectura del ecosistema, con nuevas pautas de comportamiento de los usuarios y nuevos servicios, algunos de carácter disruptivo.

Según Noam, el grueso de la acción reguladora, surgida en los años 90 del siglo pasado, y enfocada primordialmente hacia la cuestión de la competencia como motor de la innovación, está obsoleta: “Hay cuatro razones para que no resulte: inestabilidad en el sector, exigencias crecientes de inversión, economías de escala cambiantes, y presencia emergente de los medios en la industria de las telecomunicaciones" (2010, p. 5).

En el caso de los contenidos móviles se dan particularidades específicas que confieren a la función reguladora una profunda influencia sobre el desarrollo del ecosistema. Entre sus características más relevantes, destaca el hecho de tratarse de un fenómeno nuevo, porque los contenidos móviles son un producto del siglo XXI. También es importante tener presente que se trata de una actividad producto de la convergencia de dos conceptos -las redes de comunicación móvil y las redes de difusión de contenidos-, cada uno referenciado en marcos regulatorios diferentes, propios de momentos históricos y de características materiales distintas: el propio de los servicios de telecomunicaciones, heredado del entorno telefónico y en menor medida de Internet -este último, por múltiples razones, mucho menos regulado que el telefónico- y el vinculado a las diferentes ramas de las industrias de contenidos. 
La tecnología de conmutación de paquetes, cuya implementación por los gestores de redes transcurre con la llegada de las tecnologías GPRS o 2,5G, se ha desarrollado en una fase de crecimiento de Internet en la que han ido cobrando protagonismo aplicaciones y servicios -audio y video en streaming, VoIP...- en los cuales es crítica la latencia a los efectos de una calidad aceptable de la experiencia de usuario. De ese modo, los aspectos relacionados con la gestión del tráfico han cobrado un protagonismo desconocido para tiempos anteriores en los que era mayor el peso del tráfico de datos -navegación de red, intercambio de archivos, correo electrónico- que acusan en menor medida los efectos de situaciones de congestión.

Según la OCDE (2007), el tráfico se regula a partir de tres criterios generales: el del "máximo esfuerzo"2; la priorización basada en la necesidad; y la priorización activa.

En el primer caso, cuando se produce una situación de saturación en la red, no queda más remedio que desechar los paquetes más antiguos que esperan a ser direccionados en el buffer. En la aplicación del segundo criterio, una vez los operadores de redes móviles han establecido un criterio para los casos de congestión que otorga prioridad de paso a unos paquetes -VoIP o video en streaming, por ejemplo, en detrimento de correo electrónico, intercambio de archivos P2P y navegación Web, por ejemplo-, quedan establecidas calidades de servicio diferentes para unos servicios en detrimento de otros. Y en el tercer caso, el operador está facultado para establecer prioridades de paso permanentes en detrimento de ciertos tipos de paquetes, incluso en situaciones de máxima fluidez en la red.

La situación que parece prestarse a mayores dificultades de interpretación ha sido tradicionalmente la basada en el protocolo de máximo esfuerzo o best effort, en ocasiones también denominada del cartero, en la que el buen fin de la transacción no está sujeto a compromiso alguno en relación con el tiempo: si no va acompañada de algún tipo de compromiso de servicio mínimo deja al usuario en manos de la buena fe y de la capacidad de gestión de la red -en aquellos aspectos no vinculados a la gestión del tráfico- que ejerza su proveedor de telecomunicaciones.

Por ejemplo, Gamo, de Telefónica, sostiene que la desigualdad en el trato de los paquetes es inherente al funcionamiento mismo de la red: “Entre los meca-

DOI: ri14.v12i1.613 | ISSN: 1697-8293 | Año 2014 Volumen 12 Nº 1 | ICONO14 
nismos concebidos para permitir esta discriminación, podemos citar sin ánimo de exhaustividad los campos de Tipo de Servicio (TOS), la posibilidad de que distintos tráficos utilicen distintos protocolos con distinto funcionamiento y garantías (TCP y UDP, por ejemplo), los mecanismos de priorización de celdas y paquetes, los mecanismos de control de flujo, throttle ${ }^{3}$ y ventanas deslizantes, etc., etc., etc. Toda esta complejidad está prevista y es necesaria para permitir un encaminamiento y una asignación de los recursos de red eficientes y eficaces. Estos mecanismos deben ser viables económicamente y garantizar a cada usuario una serie de mínimos con independencia de lo que estén haciendo los demás usuarios. La neutralidad no es por tanto una cuestión técnica" (2007, p .1).

Esta visión conduce directamente, se quiera o no, al examen de una nueva cuestión, que no es otra que la determinación, o por lo menos el mero reconocimiento, de los mencionados "tráficos con distintas necesidades", para lo que se hace ineludible el recurso a algún tipo de procedimientos de "catalogación del tráfico" o "packet shaping" (Carter, Scott et al., 2010), empleados en la mayor parte de los operadores de redes de telecomunicaciones y conocidos y respaldados por buena parte de los reguladores del sector en La UE y Norteamérica. Este tipo de procedimientos se basan en la identificación de los diferentes tipos de datos de cada usuario, por ejemplo voz, navegación Web, correo, descargas P2P, streaming, etc.

El regulador británico Ofcom ha sido uno de los primeros en La UE a la hora de tomar conciencia de lo relevante del problema de la neutralidad, y algunos de sus documentos (Ofcom, 2010) han servido de referencia a la hora de orientar las políticas al respecto en diferentes países. Su gráfico sobre la gestión del tráfico en la red es uno de sus fragmentos más difundidos. En el área más cercana al amarillo se encuentran las prácticas menos intervencionistas, que son las denominadas de máximo esfuerzo o principio del cartero: el operador/proveedor de acceso hace todo lo que está en su mano por facilitar el flujo libre de paquetes y no discrimina entre diferentes calidades de servicio para un mismo ancho de banda contratado. Más allá de la zona netamente amarilla comienzan los escenarios de priorización del tráfico, hasta alcanzar el otro extremo, donde se encuentran prácticas lesivas a la competencia y contrarias a los derechos del cliente, tales como bloquear o restringir la circulación de contenidos de empresas rivales, incluso a cambio de alguna contraprestación económica. 


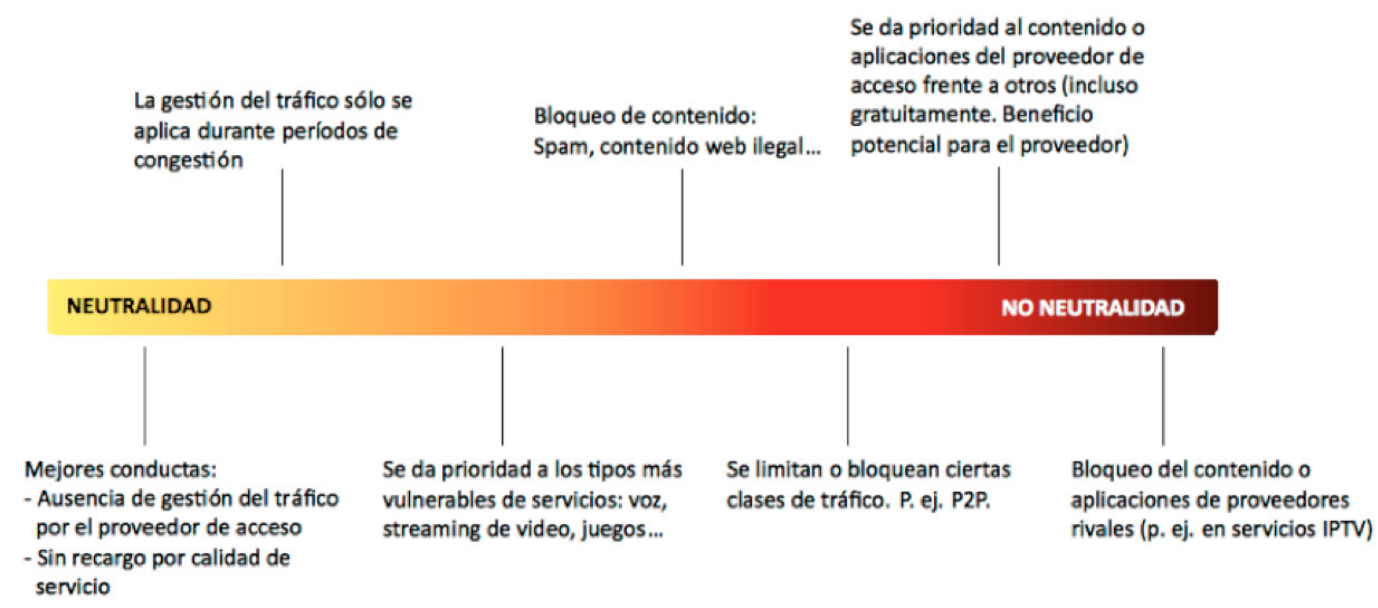

Gráfico 1. Fuente: Ofcom, 2010.

Sin embargo, mercados como el japonés han alcanzado las mayores cotas de extensión de la banda ancha fija y móvil a partir de presupuestos teóricos reguladores diferentes. Sin procedimientos de catalogación del tráfico no es posible en el actual momento de desarrollo de la tecnología ejecutar ninguna de las estrategias de priorización antes mencionadas.

La herramienta más acreditada para este fin es la tecnología Deep Packet Inspection (DPI), muy implementada en España entre los operadores de telefonía móvil, y que permite bucear en el origen y tipología de los paquetes de datos. Mediante el uso de la DPI las compañías telefónicas detectan, por ejemplo, cuándo un usuario manda mensajes breves o habla por tecnología IP. El uso de estos procedimientos suscita recelos entre reguladores europeos como el británico, que ven en peligro la privacidad de las comunicaciones (Ofcom, 2010). La opinión del Supervisor Europeo de Protección de Datos es aún más taxativa: "vulnera la neutralidad de Internet y la confidencialidad de las comunicaciones. Ello pone de relieve aspectos graves en relación con la protección de la privacidad de los usuarios y de los datos personales". (Hustinx, 2011: 20)

En España, desde la Comisión del Mercado de las Telecomunicaciones (CMT) se sugiere incluso un orden de prelación: “Según el Information Technology and In- 
novation Foundation, el orden lógico de prioridad de los paquetes debería ser: los de real-time (VoiP, gaming), streaming, navegación web y finalmente, otros como el P2P o el FTP" (CMT Blog, 2010).

No coincide esta visión oficiosa del regulador español con la posición expresada posteriormente en 2011 por el organismo que agrupa a los reguladores de los 27 miembros de la UE, el BEREC, que en su documento "Un marco para la calidad de servicio a la luz de la neutralidad de la red" prefiere que las situaciones de exceso de tráfico se gestionen "de un modo agnóstico en cuanto a la aplicación, con lo que constituyen una alternativa a la aplicación de las medidas específicas típicamente desarrolladas con DPI". (BEREC, 2011, p. 19).

\subsection{Internet de dos carriles o dos Internet}

El establecimiento de una red paralela a Internet con el fin de asegurar mejores prestaciones a un sector de usuarios es una pretensión a veces planteada en los debates sobre el futuro de la red. Se trataría, según Economides y Tåg, de "crear un 'carril especial' para los paquetes de información de firmas que pagaran al tiempo que se restringe el carril para los que no pagan, sin ampliar la capacidad total" (2007, p. 4). Estos planes han sido vistos por exponentes cualificados de los usuarios de la red como incentivadores de la degradación de la calidad del servicio. En palabras de uno de los fundadores de Google, Sergei Brin, "el único modo de que uno pueda tener un carril rápido que sea útil -es decir, que la gente pague extra por él- es que haya carriles lentos" (según cita de Murakami, 2007, p. 5).

Al tiempo, el desarrollo de la red ha dado lugar a la aparición de los llamados "servicios gestionados" (Ofcom, 2011; Vakulenko, Schuermans, Constantinou, y Kapetanakis, 2011), en los que el proveedor de un cierto servicio mediante la red, video en streaming por ejemplo, se hace responsable ante el usuario final de su desempeño en ciertas condiciones de calidad.

La relevancia de estos servicios gestionados en el desarrollo de la red en los próximos años se puede observar en el cuadro $n^{0} 2$, que expresa gráficamente las previsiones de crecimiento del tráfico de Internet para España en el período 2011-2016.

ICONO14 | Año 2014 Volumen $12 N^{\circ} 1$ | ISSN: 1697-8293 | DOl: ri14.v12i1.613 


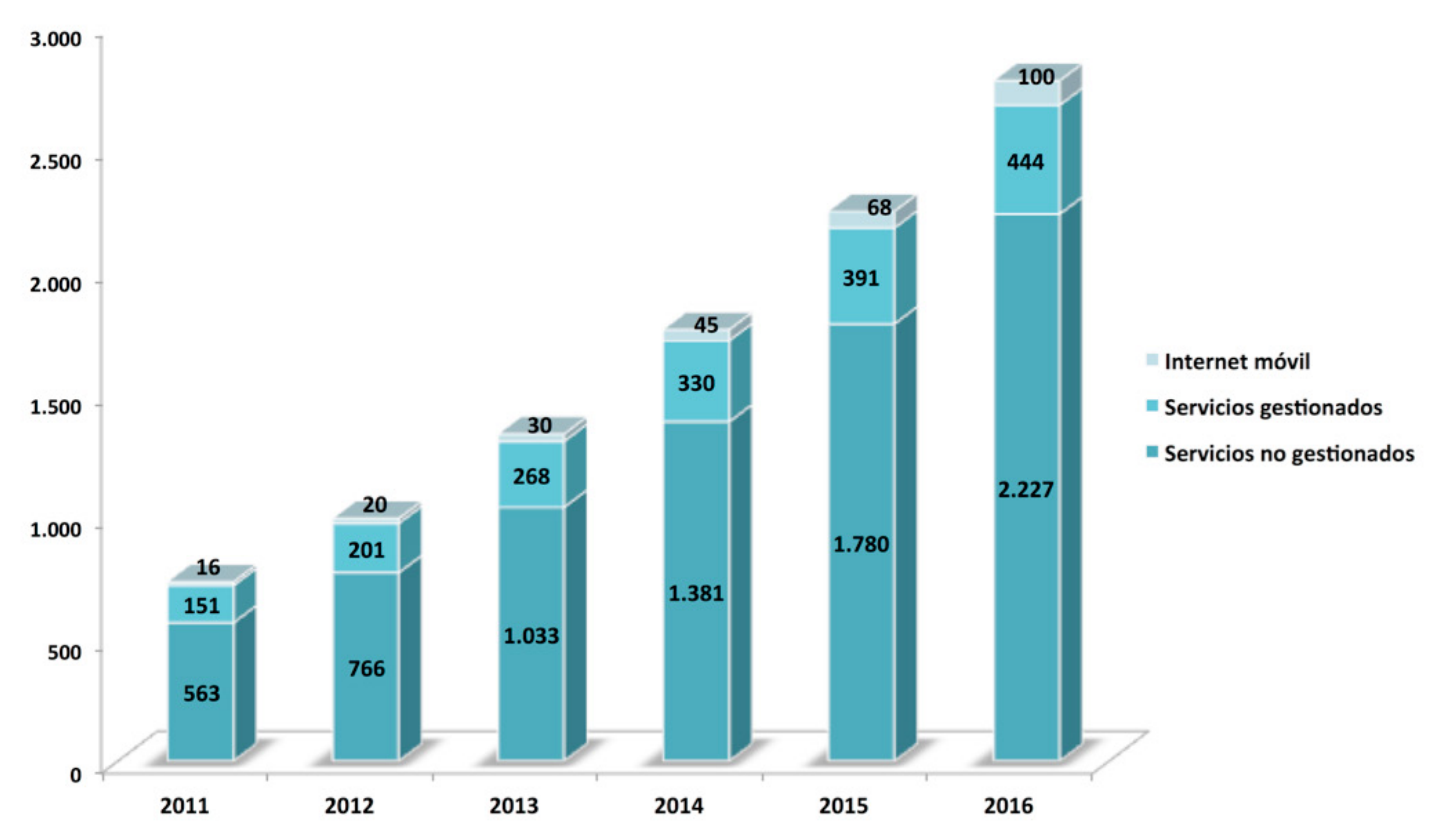

Gráfico 2. Fuente: Cisco VNI (datos obtenidos el 25/2/2013 mediante el Cisco VNI Forecast Widget disponible en http://ciscovni.com/vni_forecast/advanced.html)

Puede observarse cómo, según estas estimaciones, los servicios gestionados, que supusieron en 2012 cerca de una quinta parte de Internet en su conjunto, van a duplicar su volumen hasta 2016.

En el sector de los operadores de redes, tanto fijos como móviles, se ha propugnado tradicionalmente que esta situación de mercado bilateral debería otorgarles el derecho a cobrar, no solo a los prestadores de servicios gestionados, sino a cualquier empresa cuya actividad esté ligada a la red y la utilice intensivamente en su modelo de negocio. Las empresas operadoras de redes móviles, específicamente en España Telefónica y Vodafone (elmundo.es, 8/2/2010; 16/2/2010) han puesto siempre como ejemplo el caso de los buscadores. También en EE.UU. proveedores de servicios de telecomunicaciones como Verizon y AT\&T (Giga 0M, 2012), así como varios operadores de cable, han expresado quejas similares (Economides y Tåg, 2007). Sorprendentemente, esta posición ha sido compartida en ocasiones por 
Microsoft (Infobae, 10/10/2010), que entre sus actividades cuenta con una participación del 50\% en Bing, uno de los buscadores líderes.

Por su parte, las escasas veces que el gobierno español ha entrado en el debate ha sido para apoyar a los operadores de redes en sus reclamaciones hacia los buscadores (El Mundo, 15/2/2010).

\subsection{EEUU: origen y referencia en la regulación}

Si en la mayor parte de los negocios la regulación de la actividad es posterior al desarrollo de esta, en el caso de Internet la cuestión de la neutralidad no deja de ser un mero objeto de hipótesis hasta la aparición de los primeras denuncias de usuarios que reclaman protección de las autoridades frente a abusos de sus proveedores de servicios. Ello no ha impactado en la actividad reguladora hasta la primera década del siglo XXI, cuando las principales autoridades supervisoras del mundo -singularmente la Federal Communications Commission (FCC) de EEUU y la Comisión Europea (CE)- han adoptado sus primeras posiciones sobre el asunto.

La FCC publicó en 2005 una declaración oficial o "Policy Statement" (FCC, 2005) en la que estableció las pautas que debían guiar las actuaciones del regulador para velar por la neutralidad de la red: la libertad de acceso a contenidos; la libertad de ejecución de aplicaciones y servicios; la libertad de conexión de dispositivos; y el derecho a la libre competencia entre proveedores de red, servicios/aplicaciones y contenidos.

Puede ser relevante también observar algo que no figura en el texto, y es la falta de referencia al internet móvil en tanto que objeto diferenciado en cuanto a la aplicación de los principios y acciones de regulación de la FCC.

La aplicación práctica de estas cuatro libertades acuñadas por la FCC se somete a su primera gran prueba de fuego con el llamado Caso Comcast. En 2008 la FCC atendió las protestas de usuarios de Internet servidos por Comcast, el mayor operador de cable en EE.UU., que se descargaban archivos a través de BitTorrent, una plataforma para compartir archivos. Mientras Comcast argüía que no había bloqueado

ICONO14 | Año 2014 Volumen 12 NN$^{\circ} 1$ | ISSN: 1697-8293 | DOI: ril4.v12i1.613 
las comunicaciones de sus clientes sino que se había limitado a demorar su flujo, los abonados reclamaban que el procedimiento empleado por Comcast interrumpía sus descargas vía BitTorrent.

El acuerdo de la FCC no impuso sanción alguna pero obligó a Comcast a modificar sus prácticas de gestión de red. Este suceso está considerado como la primera acción imperativa significativa emprendida por un regulador en defensa de la neutralidad de la red.

Recientemente, una sentencia del Tribunal de Apelación de EE.UU. ha resuelto que la FCC no disponía de autoridad suficiente para haber dictado esa resolución sobre Comcast, lo que ha obligado a la FCC a reelaborar en una "tercera vía" los fundamentos jurídicos que habían orientado su actuación en los últimos años (Genachowski, 2010).. Se trata de un viejo debate (Pool, 1983), que se remonta al estudio de las bases constitucionales del país, sobre si las libertades de los viejos medios -medios escritos- y los nuevos -medios de difusión masiva y medios digitales- merecen la misma protección jurídica. En lo que respecta a internet, el debate acerca de si se trata de un "servicio de información" o de "telecomunicaciones" (Copps, 2010) -en el segundo caso los derechos de los consumidores serían tutelados directamente por la FCC- se halla en el centro de una lista cada vez más larga de resoluciones judiciales y administrativas, con los subsiguientes cambios de doctrina (Genachowski, 2010).

El siguiente paso en el desarrollo normativo estadounidense, en 2010 (FCC, 2010), viene marcado por la extensa consulta pública lanzada por la FCC en 2007 con más de 100.000 comunicaciones escritas recibidas, y la consiguiente elaboración en el seno del regulador.

A partir de una apelación al principio complementario de “gestión razonable de la red", se establecen tres normas básicas:

1. Transparencia: los proveedores de banda ancha fija y móvil deben revelar las prácticas de gestión de la red, las características de rendimiento, y los términos y condiciones de sus servicios de banda ancha.

DOI: ri14.v12i1.613 | ISSN: 1697-8293 | Año 2014 Volumen 12 Nº 1 | ICONO14 
2. No al bloqueo. Los proveedores de banda ancha fija no pueden bloquear contenido, aplicaciones o servicios legales, o dispositivos no nocivos; los proveedores de banda ancha móvil no pueden bloquear sitios Web o aplicaciones legales que compitan con sus propios servicios de telefonía de voz 0 de video.

3. No a la discriminación arbitraria. Los proveedores de banda ancha fija no pueden discriminar sin motivo mientras transmitan tráfico legal de red.

Se trata de la primera resolución normativa de la FCC sobre neutralidad de la red que incluye indicaciones específicas acerca de la conducta de los operadores de telecomunicaciones en la Internet móvil. En ella se opta por una aplicación atenuada de los tres principios para la banda ancha móvil: "A la luz de estas consideraciones, concluimos que es apropiado en este tiempo dar pasos mesurados para proteger la apertura de Internet cuando se accede a través de banda ancha móvil." (FCC, 2010, p. 54).

La inaplicabilidad de la regla de no discriminación irrazonable a las redes móviles abre una brecha para eventuales prácticas restrictivas o anticompetitivas de los operadores de redes, que dificultarían el acceso a determinados contenidos como por ejemplo video o audio en streaming, intensivos en consumo de ancho de banda. Al mismo tiempo, la FCC protege el derecho de los consumidores a utilizar servicios de telecomunicaciones, los llamados servicios OTT, que compitan con los propios de su operador de telecomunicaciones.

La FCC no se pronuncia acerca del uso de otras aplicaciones móviles más vinculadas al consumo de contenidos, como por ejemplo los servicios de música y de video en streaming no ligados a operadores de redes, que sí podrían quedar en posición de debilidad ante la posibilidad de que un operador desarrollara prácticas de gestión de tráfico orientadas a favorecer el flujo de datos de sus propios asociados.

En ese caso, la FCC, que en el caso de las redes fijas desaconseja por razones técnicas y éticas la inspección de paquetes como criterio para establecer prioridades, podría adoptar criterios parecidos para la red móvil si se plantearan conflictos en el futuro: "generalmente es preferible ni requerir ni animar a los proveedores de banda ancha a examinar el tráfico de Internet para discernir qué clase de tráfico 
se halla sujeto a las reglas. (...) Es improbable que los proveedores de banda ancha pudieran identificar con fiabilidad ese tráfico en todas las circunstancias, particularmente el tráfico de voz o video originado desde nuevos servicios que utilicen protocolos inusuales. (...) Proveedores de servicios y usuarios finales tratarían de obtener la protección de nuestras reglas disfrazando su tráfico como comunicaciones protegidas" (Íbid: 29).

Pero la acción regulatoria de la FCC en su conjunto resulta debilitada de nuevo por una resolución de la justicia. En enero de 2014 un Tribunal de Apelación ${ }^{4}$ del Distrito de Columbia ha anulado las resoluciones de la FCC de 2010 sobre la neutralidad de la red, a petición de Verizon, el principal proveedor de servicios de banda ancha del país. El impacto de la sentencia está todavía por manifestarse, aunque parece abierto el camino para que los operadores de telecomunicaciones puedan reclamar en el futuro, por lo menos a los prestadores de los servicios gestionados, algún tipo de compensación económica adicional (Moritz y Edwards, 2014). La FCC, cuyo recientemente nombrado presidente es un antiguo directivo del sector, tiene asimismo por delante la resolución del dilema de si puede y quiere elevar el asunto hasta el Tribunal Supremo, o incluso enfocar la regulación de los servicios de banda ancha en términos legalmente menos vulnerables a la luz de la legislación estadounidense.

\subsection{UE: a remolque de EEUU con sus propias complejidades añadidas}

En la Unión Europea (UE), el debate sobre la neutralidad de la red ha ido generalmente a remolque del estadounidense. No es extraño, por ello, constatar que a cada paso relevante dado por la FCC suceda alguna acción de envergadura desde el lado europeo. Las manifestaciones de las dos comisarias europeas con competencias sobre la red -Reding en 2008 y Kroes en 2010- han tomado como referencia principal los trabajos de la FCC de 2005. La convocatoria de una amplia encuesta pública en EEUU en 2007 y la consiguiente resolución de la FCC de 2010 son dos actos relevantes que tienen también reflejo posterior en la UE: en 2009 una Directiva mandata a la Comisión para lanzar una amplia consulta pública sobre el tema, y el BEREC hace lo propio en verano de 2012; a finales de ese mismo año el organismo

DOI: ri14.v12i1.613 | ISSN: 1697-8293 | Año 2014 Volumen 12 N 1 | ICONO14 
de los reguladores publica dos documentos con sus posiciones acerca de la neutralidad, y otros cuatro informes que recogen el resultado de su encuesta pública.

Así, la gestión de la neutralidad de la red es de aparición reciente entre las principales preocupaciones de la UE si atendemos a sus documentos políticos y a su actividad legislativa. En su comunicación "A Digital Agenda for Europe" (Comisión Europea, 2010) no se halla ninguna referencia a la cuestión de la neutralidad. Los primeros trazos de actividad normativa garantista con respecto a la neutralidad se remontan solo a 2009, con la Directiva 2009/140/CE. Dicha norma reforma el artículo 8.4 de la Directiva 2002/21/CE, que establece las obligaciones de las autoridades nacionales de regulación a la hora de velar por los intereses de los ciudadanos. A dicho artículo le añade el punto g, que dispone: "promoviendo la capacidad de los usuarios finales para acceder y distribuir la información o utilizar las aplicaciones y los servicios de su elección". Igualmente, en el terreno de los derechos civiles y en su aplicación a las comunicaciones electrónicas son relevantes las directivas 2002/58/CE y 2009/136/CE sobre la privacidad y las comunicaciones electrónicas.

La regulación de Internet en su vertiente de servicio de telecomunicaciones se produce como resultado de una confluencia de actuaciones: La CE fija el marco regulador de las telecomunicaciones mediante directivas que deben ser trasladadas a las 28 respectivas legislaciones nacionales; y la función regulatoria, que corresponde a ministerios y entes reguladores de los estados, que en virtud de lo dispuesto en el marco regulatorio del sector que entró en vigor en 2009 se coordinan en el Organismo de Reguladores Europeos de las Comunicaciones Electrónicas (ORECE), también conocido por sus siglas inglesas BEREC. El BEREC, que tiene condición legal de Agencia Europea, es un organismo cuyas resoluciones tienen la fuerza de una recomendación.

Entre las primeras declaraciones públicas relevantes es de destacar la de la comisaria para la sociedad de la información y los medios de comunicación, Viviane Reding, que en 2008 esbozó dos principios, de "competencia" y "transparencia" (Reding, 2008). Manifestó también un punto de vista favorable a la máxima libertad de gestión de la red para sus operadores, con algunas limitaciones: los comportamientos "abusivos o anticompetitivos que limiten la elección del consumidor" 0

ICONO14 | Año 2014 Volumen $12 N^{\circ} 1$ | ISSN: 1697-8293 | DOl: ri14.v12i1.613 
que, "en algunas situaciones, la calidad del servicios de todos los operadores podría degradarse a niveles inaceptablemente bajos" son los casos en los que el regulador, europeo o nacional, debería intervenir.

A finales de 2009 el presidente de la Comisión Europea encargó a Neelie Kroes, a la vez vicepresidenta de la Comisión, las competencias sobre la Agenda Digital de la UE. En sus declaraciones posteriores Kroes (2010) amplía el marco definido por Reding con un planteamiento más detallado. La nueva comisaria hace suyos los cuatro principios de la Declaración de 2005 de la FCC (derecho de acceso, derecho a ejecutar aplicaciones, derecho a utilizar dispositivos, y derecho a la competencia entre proveedores), más el nuevo principio de transparencia propuesto por Genachowsky en 2009. En una línea similar a la doctrina expuesta por la FCC en su Notificación de Encuesta de 2007, Kroes propone cinco criterios orientativos:

1. La libertad de expresión es fundamental.

2. La transparencia no es negociable.

3. Inversión en redes eficientes y abiertas.

4. Competencia justa.

5. Apoyo a la innovación.

De la intervención de Kroes puede desprenderse un deseo de conciliar la preservación de la neutralidad de la red y su carácter de canal de transmisión de contenidos libre con un marco legislativo que no obstaculice, cuando no proteja, la actividad de las empresas operadoras.

En este período la CE actúa por mandato de la Directiva que modifica el Marco Regulador Común de las telecomunicaciones (Directiva 2009/140/CE del Parlamento Europeo y del Consejo de 25 de noviembre de 2009), que ordena una "amplia consulta pública" sobre la regulación de internet.

La citada directiva concluye con la siguiente Declaración de la CE: “la Comisión otorga gran importancia al mantenimiento del carácter abierto y neutral de Inter-

DOI: ri14.v12i1.613 | ISSN: 1697-8293 | Año 2014 Volumen 12 Nº 1 | ICONO14 
net", una declaración política que, como no es inusual en la UE, deja a posteriores procedimientos de la Comisión, el Parlamento Europeo y los reguladores nacionales la materialización en actos de los grandes principios.

Sin embargo, el diario oficial europeo comienza a reducir la brecha que le separa de EEUU en la medida que otorga carta de naturaleza al concepto de "libertades de Internet" y se refiere a la neutralidad de la red como "objetivo político" y "principio regulador", y prescribe que ambos han de formar parte de las políticas públicas de los 27 estados miembros de la UE.

En 2011 la Comisión Europea fijó su posición mediante el documento "El Internet abierto y la neutralidad de la red en Europa" (Comisión Europea, 19/4/2011), en la que respalda la idoneidad del actual Marco Regulador como base normativa para trabajar por la neutralidad e indica una cierta preferencia hacia las medidas nacionales que establezcan niveles de calidad de servicio en virtud de la Directiva de Servicio Universal: "tienen el poder de intervenir estableciendo obligaciones de calidad mínima de servicio para los servicios de redes de transmisión" (Íbid., p. 5).

La CE admite que el grueso del debate sobre la neutralidad se organiza en torno a la gestión del tráfico, y se compromete a trabajar estrechamente con el BEREC para monitorizar la situación y adoptar medidas en caso necesario.

En cuanto a la dualidad fijo/móvil, la Comisión no se define a la hora de pronunciarse acerca de si debe adoptarse un tratamiento específico en la gestión del tráfico en Internet móvil.

La resolución del Parlamento Europeo sobre la neutralidad de la red de 2011 (Parlamento Europeo, 17/11/2011), insta a la Comisión a cooperar con el BEREC en la vigilancia de las prácticas de gestión del tráfico y a promover la transparencia de los operadores en la relación con sus clientes, siempre partiendo de la premisa de que "no existe en las circunstancias actuales una necesidad clara de intervención reguladora adicional" (punto 2). 
El BEREC, que no mantuvo su primera reunión hasta enero de 2010, presentó en 2011 un primer documento en el que explora las implicaciones del objetivo de neutralidad de la red en relación con la calidad de servicio. Hasta finales de 2012 este organismo de los reguladores europeos no fija sus directrices. La doctrina del BEREC está sintetizada en el documento "Resumen de las posiciones del BEREC sobre la neutralidad de la red" (BEREC, 2012a); el informe “Perspectiva general de la aproximación del BEREC a la neutralidad de la red" (BEREC, 2012b), por su parte, refleja los puntos de partida conceptuales del primer documento $\mathrm{y}$, aunque no está calificado como tal, podría interpretarse como preámbulo del mismo.

En su compendio de directrices, la agencia que agrupa a los reguladores de la UE parte de la idea de que la neutralidad de la red se verifica principalmente en lo que atañe a la gestión del tráfico en la red: "se considera improbable una violación del principio de neutralidad de la red si todo el tráfico se trata sobre la base del mayor esfuerzo" (BEREC, 2012a, p. 1). Por ello considera esencial la fijación de los criterios que han de presidir la actuación de los proveedores de acceso a Internet, y que son los siguientes:

1. No discriminación entre actores.

2. Control en manos del usuario final

3. Eficiencia y proporcionalidad

4. Agnosticismo en cuanto a la aplicación.

Todo ello se plantea en un marco conceptual en el que se reconoce que “los mismos principios deberían aplicarse a las redes fijas y a las móviles, aunque el análisis caso por caso podría reflejar diferencias en condicionantes tecnológicos" (Íbid, p. 7). En ello los reguladores europeos coinciden con los criterios formulados en diferentes reuniones de la Unión Internacional de Telecomunicaciones, en los que es habitual hallar declaraciones de contenido similar: "el principio que informa la neutralidad de la red tiene que ser el mismo para las redes fijas y móviles, incluso si las herramientas utilizadas para la gestión de dichas redes son diferentes" (ITU, 2012, p. 6).

DOI: ri14.v12i1.613 | ISSN: 1697-8293 | Año 2014 Volumen 12 Nº 1 | ICONO14 
Por parte de los reguladores europeos se puntualiza: "existen otros aspectos (...), tales como asuntos vinculados con la preservación de las libertades públicas (como libertad de expresión o el acceso a ciertos tipos de contenido de utilidad social), así como la necesidad de mantener el orden público y la seguridad nacional (...). Aunque estos asuntos son de la mayor importancia en todas las democracias, estas consideraciones no son el foco de la labor de BEREC, ya que, en su mayor parte, se hallan fuera del mandato de los NRA. Deberían ser abordados a la luz de los marcos legislativos nacionales y europeos relevantes" (BEREC, 2012a, p. 10). De este medo sitúan su labor en un terreno no convergente, circunscrito a la regulación estricta del servicio de telecomunicaciones. No es el caso de reguladores como el británico, por ejemplo, donde el organismo regulador Ofcom dispone de competencias en materia de contenidos (Communications Act, 2003).

La comparación entre los marcos normativos europeo y estadounidense remite a una primera consideración: mientras que la FCC plantea obligaciones a los proveedores de servicios ("los proveedores deben..."), el BEREC "propone (...) criterios de evaluación" para "evaluar la razonabilidad de las prácticas de gestión del tráfico" (BEREC, 2012a, p.6), lo que conecta con el principio de "gestión razonable" de la FCC. Si hay que acudir a la literalidad de lo expresado en los documentos de unos y otros, ambos coinciden en oponerse a la discriminación injustificada; y donde en EEUU se habla de "no al bloqueo" (aplicaciones, contenidos, dispositivos...) y "transparencia" (prácticas de gestión de la red), en la UE se proclama la necesidad de "control en manos del usuario final" (criterios de gestión del tráfico, transparencia), y "eficiencia y proporcionalidad" (gestión del tráfico).

En cuanto a la dicotomía Internet fijo/móvil, mientras que en EEUU la FCC solo aspira a imponer "transparencia" y "no al bloqueo" a los operadores móviles, en La UE sí se aplican los mismos principios para todas la red, aunque cabe la posibilidad de herramientas distintas en su aplicación.

\subsection{Japón: otro mercado, otras soluciones}

El caso de Japón pone de manifiesto otro modo de abordar la cuestión de la neutralidad, con una visión menos imperativa en cuanto a la normativa, aunque 
no por ello pobre en cuanto a resultados: “El abordaje japonés hasta ahora se caracteriza por la carencia de reglas formales y específicas y por la amplia autoridad garantizada por la ley al Ministerio de Asuntos y Comunicaciones Interiores" (Carter, Marcus, et al., 2010: 13). Tal vez haya sido esta falta de recurso a normas explícitas lo que haya impedido la difusión del modelo entre las tradiciones europea y norteamericana, de raíz más formalista.

Su punto de partida normativo se halla en la Ley del Negocio de las Telecomunicaciones, de 2007, que en su artículo 6 prescribe que "ningún proveedor de telecomunicaciones deberá incurrir en trato injusto y discriminatorio en la provisión de servicios de telecomunicaciones" (Ministry of Internal Affairs and Communications, 2007).

En 2008 el ministerio japonés promulgó una serie de "pautas" (Carter, Marcus et al., 2010) que, sin ser de obligado cumplimiento, sí afectan al conjunto del sector de Internet. En ellas se establece que las prácticas de catalogación de tráfico, en tanto que probablemente atentatorias contra el secreto de las comunicaciones, se hallan fuera de la ley. Los procedimientos de estrangulamiento o throttling de tráfico solo podían ser usados excepcionalmente, y nunca debían sustituir a la mejora de la capacidad de la red, que se establecía como la solución a aplicar en términos generales. Aunque no se decía explícitamente, las normas eran de aplicación igualmente para redes fijas y móviles (Íbid.: 17).

En otra muestra de las peculiaridades del modelo japonés, el supervisor plantea la necesidad de discutir la asignación de los costes derivados de la mejora de las redes: "el Ministerio puede reexaminar la economía del mercado de la banda ancha con el fin de determinar cómo están asignados los costes a lo largo del ecosistema de la banda ancha con el fin de comprender si, por ejemplo, los proveedores de contenidos son agentes que se benefician sin contribuir ${ }^{5}$ o si colaboran equitativamente en los costes del sistema en su conjunto" (Carter, Marcus et al., 2010: 18).

De esta observación se desprende la posibilidad de que, como consecuencia de este estudio, proveedores y/o agregadores de contenidos se vean compelidos a pagar por la circulación de sus contenidos en la red, algo que como ya se ha relatado 
antes en este mismo capítulo pretenden en La UE y en Norteamérica los operadores de las redes que albergan a Internet.

Es necesario tener en cuenta, sin embargo, que las provisiones regulatorias japonesas no han sido contrastadas con una inspección a conciencia ni con una vigilancia social como la que puede encontrarse en sociedades más movilizadas como por ejemplo la estadounidense. A ello se suma el hecho de que el mercado japonés de servicios de telecomunicaciones está organizado en torno a la fuerte presencia de NTT DOCOM0, lo que en determinados territorios limita la competencia tal como se entiende en La UE y América.

\section{Discusión y conclusiones}

Tras años en que el debate acerca de la neutralidad se ofrecía como un asunto hipotético, los últimos documentos del BEREC confirman que hemos entrado en una etapa en que es necesario hallar soluciones para problemas reales, que están presentes por lo menos en un $20 \%$ de los casos. Según los reguladores europeos las vulneraciones se dan principalmente en los servicios 0TT de voz y de mensajería instantánea. Ello se debe no solo a la creciente popularidad de estos servicios, sino también al poder de lobby de las empresas que los ponen en el mercado, y que pueden hacer oír con claridad su voz en los foros europeos donde afloran estas quejas. Resulta mucho más difícil, por tanto, que los usuarios individualmente o en pequeñas agrupaciones hagan llegar sus quejas hasta los reguladores en un modo técnicamente solvente, por lo que no disponemos de datos realmente solventes capaces de indicar hasta qué punto se producen restricciones significativas a la circulación de otros contenidos, como por ejemplo video en streaming o servicios P2P.

De todo lo expuesto anteriormente se desprende que el debate sobre la neutralidad de la red se ha convertido en un debate sobre los sistemas de ordenación del tráfico de paquetes en situaciones de congestión, y las diferentes soluciones técnicas para abordarlo. Los organismos encargados de la regulación en La UE, EEUU y Japón coinciden, con matices, en la conveniencia de evitar los procedimientos más intrusivos, tipo DPI, y tender a herramientas que anonimicen a usuarios y a contenidos.

ICONO14 | Año 2014 Volumen $12 N^{\circ} 1$ | ISSN: 1697-8293 | DOI: ri14.v12i1.613 
Con ser este el más candente de los aspectos prácticos relacionados con la neutralidad, no es el único. Los reguladores eluden pronunciarse acerca del efecto de propuestas como la introducción de tarifas adicionales a desarrolladores o a buscadores por el lucro obtenido mediante la red. En el caso europeo particularmente se entiende que este aspecto pertenece a los gobiernos nacionales, la CE o el PE, que según el BEREC serían los encargados de velar por las libertades de Internet.

Ninguna autoridad nacional e internacional ha desarrollado por el momento criterios detallados acerca del papel que se otorga a los llamados servicios gestionados y al modo en que los reguladores deben garantizar los derechos de usuarios y proveedores de contenidos en una Internet de dos carriles. Tampoco se ha desarrollado si esta vía a doble carril estaría seccionada por medianas infranqueables o si, por el contrario, podría estructurarse con líneas discontinuas que permitieran una gestión flexible de las capacidades.

La aplicación de los principios de neutralidad a la red móvil es objeto de interés creciente por parte de legisladores y reguladores. En el caso europeo, el BEREC ha promulgado una lista de criterios redactada de manera que puede aplicarse tanto a la red fija como a la móvil. El desarrollo acelerado del mercado de datos móviles dará lugar sin duda a situaciones futuras que obligarán a desarrollar este sector de la actividad reguladora.

El debate sobre la neutralidad, y sobre sus resultados normativos y de supervisión, se desarrolla muy condicionado por las presiones de los diferentes grupos de interés. Así, a las tradicionalmente poderosas compañías de telecomunicaciones herederas del mundo telefónico, se suman ahora las voces de los operadores de servicios de telefonía IP, que en razón de su volumen empresarial son capaces de hacer llegar sus propuestas a todos los niveles del debate, lo que se refleja en las diferentes resoluciones de los poderes públicos. Por el contrario, usuarios y proveedores de contenidos no vinculados con los gestores de redes no hallan sus puntos de vista recogidos con la misma fidelidad por esa falta de gestiones de lobby.

En conexión con todo lo anterior, y a resultas de la reciente sentencia del Tribunal de apelación de la ciudad de Washington, no sería inimaginable que desde los 
diferentes grupos empresariales que gestionan las redes de banda ancha en la UE se planteara a medio plazo la necesidad de acomodar la acción regulatoria europea a estándares parecidos a los de EEUU, con la pretensión de proteger al sector de un eventual déficit de competitividad con respecto a los operadores estadounidenses, lo que podría tener consecuencias, todavía no previsibles, sobre los consumidores finales y sobre los proveedores de servicios de contenidos de valor añadido independientes de los operadores de telecomunicaciones.

\section{Notas}

[1] Se entiende por mercado "abierto" aquel que pertenece a la OCDE.

[2] "Best effort" en el original.

[3] Literalmente "estrangular".

[4] Los Tribunales de Apelación de EEUU suelen tener jurisdicción pluriestatal, y no siempre sus resoluciones pueden ser objeto de apelación ante el Tribunal Supremo Federal.

[5] "Free riders" en el original.

\section{Referencias}

ARCEP. (2010). Neutralité des réseaux. Les actes du colloque. Les Rapports de l'ARCEP. Consultado en http://stakeholders.ofcom.org.uk/binaries/ consultations/net-neutrality/summary/netneutrality.pdf

Benkler, Y. (2011). Open wireless vs. Licensed spectrum: Evidence from market adoption. Consultado en http://www.benkler.org/Open_Wireless_V_Licensed_ Spectrum_Market_Adoption_current.pdf

BEREC. (2012a). Overview of BEREC's approach to net neutrality. BoR (12) 140. Consultado en http://www.berec.europa.eu/eng/news_consultations/whats_ new/1281-berec-has-adopted-two-summaries-and-the-updated-reports-onnet-neutrality

BEREC. (2012b). Summary of BEREC positions on net neutrality . BoR (12) 146 . Consultado en http://www.berec.europa.eu/eng/news_consultations/whats_ new/1281-berec-has-adopted-two-summaries-and-the-updated-reports-onnet-neutrality

Berners-Lee, T. (2006). Net neutrality: This is serious. Decentralized information

ICONO14 | Año 2014 Volumen $12 N^{\circ} 1$ | ISSN: 1697-8293 | DOI: ri14.v12i1.613 
group (DIG) breadcrumbs [Página Web]. Consultado en http://dig.csail.mit. edu/breadcrumbs/node/144

Bustamante, E. (2011). España y Latinoamérica. Economía creativa del entretenimiento digital. En Industrias creativas. Amenazas sobre la cultura digital (pp. 117-144). Barcelona: Gedisa.

Carter, K. R.; Marcus, J.; Peake, A. J.; y Watanabe, T. (2010). A comparison of network neutrality approaches in: The US, Japan, and the European Union. (38th Annual Telecommunication Policy Research Conf). Washington, DC. Consultado en http://kennethrcarter.com/vita/pubs/Net_Neutrality_in_US_ JP_EU.pdf

Castellet, A.; y Aguado, J. M. (2010). Neutralidad de la red y contenidos digitales: Conflictos de intereses y escenarios de futuro. [XXV Congreso Internacional de Comunicación. Modelos de negocio para una economía digital, el valor de los contenidos] Pamplona: Universidad de Navarra.

Castells, M. (2009). Comunicación y poder. Madrid: Alianza Editorial. Crusafon Baqués, C. (2012). La nueva era mediática: Las claves del escenario global. Barcelona: Bosch.

Cheng, H. K.; Bandyopdhyay, S.; y Guo, H. (2011). The debate on net neutrality: A policy perspective. Information Systems Research, (Web version). doi:10.1287/isre.1090.0257

Cisco. (2010). Cisco visual networking index: Usage. Consultado en http://www. cisco.com/en/US/solutions/collateral/ns341/ns525/ns537/ns705/Cisco_ VNI_Usage_WP.pdf

Clyburn, M. (2010). Remarks at "The future of the Internet" public hearingMinneapolis, Minnesota. FCC. Consultado en http://www.fcc.gov/Daily_ Releases/Daily_Business/2010/db0820/DOC-301051A1.pdf

CMT Blog. (2010). La Comisión Europea y la Net Neutrality. [Página Web] Consultado en http://blogcmt.com/2010/07/08/la-comision-europea-y-laneutralidad-de-la-red/

Comisión Europea. (2010). A Digital Agenda for Europe [Comunicación de la Comisión al Parlamento Europeo, el Consejo, el Copnsejo Económico y Social Europeo y el Comité de las Regiones] (Comunicación de la Comisión al Parlamento Europeo, el Consejo, el Copnsejo Económico y Social Europeo y el Comité de las Regiones). Bruselas. Consultado en http://ec.europa. 
eu/information_society/digital-agenda/documents/digital-agendacommunication-en.pdf

Comisión Europea. (2011). The Open Internet and net neutrality in Europe.

(Comunicación). Bruselas. Consultado en http://ec.europa.eu/information_ society/policy/ecomm/doc/library/communications_reports/netneutrality/ comm-19042011.pdf

Communications Act. (2003). Consultado en http://www.legislation.gov.uk

Copps, Michael J. (2010). Statement at "The future of the Internet" public hearing- Minneapolis, Minnesota. FCC. Consultado en http://hraunfoss.fcc. gov/edocs_public/attachmatch/D0C-301019A1.pdf

Copps, Michael J. (2003). The beginning of the end of the Internet?

Discrimination, closed networks, and the future of cyberspace. (Intervencion ante la New American Foundation). Consultado en http://hraunfoss.fcc.gov/ edocs_public/attachmatch/D0C-239800A1.pdf

Diario Oficial de las Comunidades Europeas. (2002a). Directiva 2002/21/CE del Parlamento Europeo y del Consejo de 7 de marzo de 2002 relativa a un marco regulador común de las redes y los servicios de comunicaciones electrónicas (directiva marco). Consultado en http://ec.europa.eu/information_society/ topics/telecoms/regulatory/new_rf/documents/l_10820020424es00330050. pdf

Diario Oficial de las Comunidades Europeas, C. (2002b). Directiva 2002/58/CE del Parlamento Europeo y del Consejo de 12 de julio de 2002. Consultado en Google Scholar.

Diario Oficial de la Unión Europea. (2009a). Directiva 2009/136/CE del Parlamento Europeo y del Consejo de 25 de noviembre de 2009 por la que se modifican la Directiva 2002/22/CE relativa al servicio universal y los derechos de los usuarios en relación con las redes y los servicios de comunicaciones electrónicas.

Diario Oficial de la Unión Europea. (2009b). Directiva 2009/140/CE del Parlamento Europeo y del Consejo de 25 de noviembre de 2009. Consultado en http://eur-lex.europa.eu/LexUriServ/LexUriServ.do?uri=0J:L:2009:337:00 37:0069:ES:PDF

Economides, N. y Tåg, J. (2007). Net Neutrality on the Internet: A two-sided market analysis. SSRN ELibrary. Consultado en http://archive.nyu.edu/fda/ 
bitstream/2451/26057/2/07-27.pdf

El Mundo. (2010). Miguel Sebastián apoya que Telefónica cobre un peaje

a Google. [Página Web]. Consultado en http://www.elmundo.es/

elmundo/2010/02/15/navegante/1266235275.html

FCC. (2005). FCC 05-151.(Policy Statement). Consultado en http://hraunfoss.fcc. gov/edocs_public/attachmatch/FCC-05-151A1.pdf

FCC. (2010). In the matter of preserving the 0pen Internet. Broadband industry practices. (Report and Order). Washington, DC. Consultado en http:// hraunfoss.fcc.gov/edocs_public/attachmatch/FCC-10-201A1.pdf

Feijóo, C.; Gómez-Barroso, J. L.; y Martínez, I. (2010). Nuevas vías para la comunicación empresarial: Publicidad en el móvil. El Profesional de la Informacion, 19(2), 140-148. doi:10.3145/epi.2010.mar.04

Fransman, M. (2000). Evolution of the telecommunications industry into the Internet age. Consultado en http://www.telecomvisions.com/articles/pdf/ FransmanTelecomsHistory.pdf

Genachowsky, J. (2010). The third way: A narrowly tailored broadband framework. FCC. Consultado en http://hraunfoss.fcc.gov/edocs_public/ attachmatch/DOC-297944A1.pdf

Giga 0M. (2012). AT\&T's mad, mad plan to charge wireless app developers. [Página Web] Consultado en http://gigaom.com/broadband/atts-madmad-plan-to-charge-wireless-app-developers/?utm_source=social\&utm_ medium=twitter\&utm_campaign=gigaom

Hustinx, P. (2011). Opinion of the European Data Protection Supervisor on Net Neutrality, traffic management and the protection of privacy and personal data. Consultado en http://www.edps.europa.eu/EDPSWEB/webdav/site/ mySite/shared/Documents/Consultation/Opinions/2011/11-10-07_Net_ neutrality_EN.pdf

Infobae.com. (2010). “Que cobren, pero que inviertan en la red”. [Página Web] Consultado en http://www.infobae.com/tecnologia/541144-101457-0-Quecobren-pero-que-inviertan-la-red

ITU. (2012). 12TH Global Symposium for Regulators (GSR). Chairman's Report. Colombo (Sri Lanka). Consultado en http://www.itu.int/ITU-D/treg/Events/ Seminars/GSR/GSR12/documents/GSR12_ChairmanReport.pdf Jaokar, A. y Gatti, A. (2009). Open mobile. Londres: Futuretext.

DOI: ri14.v12i1.613 | ISSN: 1697-8293 | Año 2014 Volumen 12 Nº 1 | ICONO14 
Kroes, N. (2010). Net Neutrality in Europe. Paris. Consultado en http://europa. $\mathrm{eu} / \mathrm{rapid} /$ pressReleasesAction.do?reference=SPEECH/10/153\&format=HTML\&a ged=0\&language $=E N \&$ guiLanguage $=e n$

Lessig, L. (1999). The Law of the Horse: What cyberlaw might teach. Harvard Law Review, 113(2), 501-49. Consultado en http://www.jstor.org/stable/1342331.

Lessig, L. (2009). El código 2.0. Madrid: Traficantes de sueños. Consultado en http://www.customerhunt.net/libros/codigo-2-0-lawrence-lessig.pdf Ministry of Internal Affairs and Communications. (2007). Telecommunications Business Act. Tokyo. Consultado en http://www.soumu.go.jp/main_sosiki/ joho_tsusin/eng/Resources/laws/pdf/090204_2.pdf

Moritz, S., y Edwards, C. (2014). Verizon's victory over FCC rules seen as a loss for Netflix. Bloomberg [Página Web]. Consultado en http://www.bloomberg.com/ news/2014-01-14/verizon-victory-on-net-neutrality-rules-seen-as-loss-fornetflix.html

Murakami, N. (2007). Network Neutrality in Japan. (Presentación de Norio Murakami VP and General Manager Google Japan). Consultado en http:// www.soumu.go.jp/main_sosiki/joho_tsusin/policyreports/chousa/network_ churitsu/pdf/070228_si4_7.pdf

Noam, E. (2001). Interconnection as the key to the Network of Networks. En Interconnecting the Network of Networks (pp. 1-16). The MIT Press.

Noam, E. (2010). Regulation 3.0 for telecom 3.0. Telecommunications Policy, 34(1-2), 4-10. doi:10.1016/j.telpol.2009.11.004

OCDE. (2007). Internet traffic priorisation: An overview. Consultado en http:// www.oecd.org/dataoecd/43/63/38405781.pdf

Odlyzko, A. (2009). Network neutrality, search neutrality, and the neverending conflict between efficiency and fairness in markets. Review of Network Economics, 8(1), 40-60. Consultado en http://www.dtc.umn. edu/\%7Eodlyzko/doc/net.neutrality.pdf

Ofcom. (2011). Ofcom's approach to net neutrality. Consultado en http:// stakeholders.ofcom.org.uk/binaries/consultations/net-neutrality/statement/ statement.pdf

Parlamento Europeo. (2011a). Network neutrality: Challenges and responses in the EU and in the U.S. Policy Department A: Economic and Scientific Policy. Consultado en http://www.europarl.europa.eu/document/activities/cont/20 
1105/20110523ATT20073/20110523ATT20073EN.pdf

Parlamento Europeo. (2011b). Propuesta de resolución sobre la internet abierta y la neutralidad de la red en Europa - B7-0572/2011. 2011b [Página Web]. Consultado en http://www.europarl.europa.eu/sides/getDoc.do?pubRef=-// EP//TEXT+MOTION+B7-2011-0572+0+DOC+XML+V0//ES

Pool, I. d. S. (1983). Technologies of Freedom (pp. 0-292). Cambridge, MS: Belknap Pr.

Reding, V. (2008). Net Neutrality and Open Networks - towards a European approach. (Conferencia 'Network Neutrality - Implications for Innovation and Business Online' ). Consultado en http://europa.eu/rapid/ pressReleasesAction.do?reference=SPEECH/08/473\&format=HTML\&aged=0\&la nguage $=E N \&$ guiLanguage $=n l$

Vaidhyanathan, S. (2011). The googlization of everything: And why we should worry. Berkeley \& Los Angeles, CA: University of California Press.

Vakulenko, M.; Schuermans, S.; Constantinou, A.; y Kapetanakis, M. (2011). Mobile platforms: The clash of ecosystems. Vision Mobile. Consultado en http://www.visionmobile.com/rsc/researchreports/VisionMobile-Clash-ofEcosystems_v1.pdf

Vesa, J. (2006). The role of regulation in the evolution of mobile services industry. In I2010: Comments and contributions. Eurocpr 2006: Selected papers (pp. 34-70). ENCIP.

Vesa, J. (2007). The war of the mobile and Internet worlds: Regulations and corporate players. Management Online Review. Consultado en http://www. notinnovatedhere.fi/media/download_gallery/Vesa_2007_Internet-vs-mobileworld_MORE.pdf

Wu, T. (2004). The broadband debate, a user's guide. Journal on Telecommunications \& High Techology Law, 3(1), 69-95. Consultado en http:// scrawford.net/courses/wu-one-rule.pdf

Wu, T. (2011). The master switch. The rise and fall of information empires. London: Atlantic Books. 